\title{
Vom Nutzen der europäischen Ärzteorganisationen
}

\section{Pierre-François Cuénoud}

Dr. med., Vizepräsident der FMH, Departementsverantwortlicher Tarife und Gesundheitsökonomie Spitalärzte

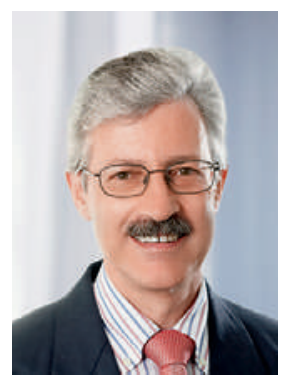

In den letzten 50 Jahren haben die europäischen Ärzteorganisationen eine beachtliche Entwicklung erfahren, angetrieben durch den Wunsch der darin aktiven Kolleginnen und Kollegen, Fortschritte zu erzielen und sich bei den Entscheidungsträgern Gehör zu verschaffen. Erfreulicherweise hat sich die Schweiz schon früh an diesem Prozess beteiligt und regelmässig Delegierte in die entsprechenden Gremien entsandt.

Einer dieser übernationalen Verbände, die Europäische Vereinigung der Fachärzte (UEMS), hat sich beispielsweise die Vereinheitlichung von Verfahren, Ausbildung und Diplomen auf die Fahnen geschrieben. Ihr ist die Einrichtung zahlreicher anerkannter europäischer Titel zu verdanken, die von den einzelnen Ländern umstandslos übernommen werden können. Die Koordinierung der Anforderungen in der Weiterbildung funktioniert inzwischen gut; Sorgen bereitet hingegen weiterhin die universitäre Ausbildung, während der gewisse Studierende immer noch keinerlei Patientenkontakt haben.

\section{Die Nachwuchsförderung muss gesichert} werden, indem genügend Geldmittel für die Aus- und Weiterbildung bereitgestellt werden.

Andere Verbände, wie der ständige Ausschuss der europäischen Ärztinnen und Ärzte (CPME) oder die Europäische Vereinigung der leitenden Krankenhausärzte (AEMH), sind grundsätzlich politisch ausgerichtet. Sie versuchen, zu den wichtigen gesellschaftlichen Entwicklungen unserer Zeit rationale medizinische Standpunkte zu erarbeiten. Unsere Gesundheitssysteme unterliegen seit jeher einem empfindlichen Gleichgewicht zwischen Liberalismus und Dirigismus, zwischen mutualistischer Subsidiarität nach Bismarck und sozialer Absicherung durch den Staat nach Beveridge.

In einigen europäischen Ländern und besonders in Osteuropa ist die Situation für die Ärzteschaft besorgniserregend: Unsere Kolleginnen und Kollegen dort arbeiten unter sehr schlechten Bedingungen und für ein miserables Gehalt; sie werden vom jeweils herrschenden politischen System stiefmütterlich behandelt und instrumentalisiert. Viele emigrieren und hinterlassen eine Lücke, die die nachrückende Generation nicht mehr auszufüllen vermag. Hier könnten die europäischen Ärzte- organisationen durch solidarisches Handeln Druck ausüben, um eine Besserung herbeizuführen.

Im Spitalbereich ist die Ärzteschaft in der Führung insgesamt noch unzureichend vertreten, obwohl empirisch bewiesen ist, dass Einrichtungen, in deren Leitungsgremien mehr Ärzte sitzen, eine höhere Qualität, Patientenzufriedenheit und Rentabilität vorweisen können. Auch in unserem Land bestreiten gewisse Autoritäten diesen Umstand beharrlich und verbannen die medizinisch-pflegerischen Verantwortlichen aus den Spitaldirektionen.

In den kommenden Jahren müssen die europäischen Ärzteorganisationen sich mehreren grossen Herausforderungen stellen:

- Die gesundheitliche Versorgung der Migrantenströme muss verbessert und koordiniert werden.

- Die verfügbaren Ressourcen werden immer knapper, was eine Medizin der zwei Geschwindigkeiten und ein entsprechendes Korruptionsrisiko zur Folge hat: Die Ärzteschaft muss sich entsprechend zur Wehr setzen.

- Angesichts des Ärztemangels werden immer mehr unserer Tätigkeiten von anderen Berufsgruppen wie Pflegepersonal oder Apothekern übernommen. Das Primat der ärztlichen Verantwortung bei der Diagnose und der Behandlung der Patienten muss gewahrt bleiben.

- Die Nachwuchsförderung muss gesichert werden, indem genügend Geldmittel für die Aus- und Weiterbildung bereitgestellt werden.

- Den präventiven Aspekten der Medizin muss zu neuem Ansehen verholfen werden.

Trotz einer allgemeinen isolationistischen Tendenz können die europäischen Ärzteorganisationen bei der Umsetzung dieser Ziele auf die aktive Unterstützung der Schweiz zählen. Unsere Stellungnahmen finden Achtung und Beachtung: Es handelt sich um einen der wenigen Bereiche, in denen die Schweiz die Prozesse der Europäischen Gemeinschaft von innen heraus beeinflussen kann. Ausserdem hilft uns der Vergleich mit unseren Nachbarn, unsere eigenen Probleme besser einzuordnen, was unsere internen Debatten bereichert und unsere Glaubwürdigkeit stärkt. 